Spoken sessions

\begin{tabular}{llllllll}
\multicolumn{6}{l}{ Abstract S127 Table 1. Apoptosis induced by recombinant TRAIL and MSC-TRAIL on combination with Vorinostat } \\
\hline & rTRAIL & Vorinostat & $\begin{array}{l}\text { rTRAIL and } \\
\text { Vorinostat }\end{array}$ & $\begin{array}{l}\text { MSCTRAIL } \\
\text { not activated }\end{array}$ & $\begin{array}{l}\text { MSCTRAIL } \\
\text { Activated }\end{array}$ & $\begin{array}{l}\text { MSCTRAIL not } \\
\text { Activated + Vorinostat }\end{array}$ & $\begin{array}{l}\text { MSCTRAIL } \\
\text { Activated + Vorinostat }\end{array}$ \\
\hline Ju77 & $7.71 \%$ & $51.35 \%$ & $80.77 \%$ & $10.32 \%$ & $48.73 \%$ & $47.44 \% \%$ & $77.7 \%$ \\
CRL2081 & $56.75 \%$ & $78.95 \%$ & $96.6 \%$ & $37.3 \%$ & $57.63 \%$ & $81.45 \%$ & $90.93 \%$ \\
One58 & $13.41 \%$ & $43.97 \%$ & $79.27 \%$ & $10.88 \%$ & $53.8 \%$ & $49.25 \%$ & $77.8 \%$ \\
\hline
\end{tabular}

MPM cells are treated with recombinant Trail $(100 \mathrm{ng} / \mathrm{ml})$ and Vorinostat $(2.5 \mu \mathrm{M})$. MSC are plated in 1:1 ratio with tumour cells. MSC are activated with doxycycline to induce TRAlL expression.

Introduction Malignant pleural mesothelioma (MPM) is a highly aggressive, incurable, chemoresistant tumour. Recent studies have shown that Mesenchymal stem cells (MSC) can home to and incorporate into the tumour stroma. Their tumour tropism can be used to deliver Tumour necrosis factor related apoptosis inducing ligand (TRAIL), a transmembrane protein that selectively induces apoptosis in transformed cells. However, not all tumours are sensitive to TRAIL. TRAIL works through triggering the extrinsic apoptotic pathway while conventional chemotherapeutic agents act by triggering the intrinsic apoptotic pathway. We hypothesised the crosstalk between these two pathways could be exploited by combining chemotherapy and MSC-TRAIL in MPM tumour cell lines.

Methods MSC were engineered to express TRAIL using a lentiviral plasmid vector. A Tetracycline (Tet)-inducible system was used as a backbone to control the expression of TRAIL. Apoptosis induced by recombinant TRAIL, MSC-TRAIL in MPM cell lines on combination with Vorinostat, a chemotherapeutic agent, was measured by Annexin-V/DAPI based flow cytometry.

Results The combination of recombinant TRAIL and Vorinostat act synergistically to induce apoptosis in MPM cell lines. Recombinant TRAIL and Vorinostat, as monotherapies induce $7.17 \%$ and $51.35 \%$ apoptosis in an MPM cell line JU77 respectively. In CRL2081 and ONE58 cell lines, recombinant TRAIL induces $56.75 \%$ and $13.41 \%$ apoptosis while Vorinostat leads to $78.95 \%$ and $43.97 \%$ apoptosis respectively. The combination of recombinant TRAIL and Vorinostat shows an increased amount of apoptosis in JU77, CRL2081 and ONE58 cell lines at $80.77 \%, 96.6 \%$ and $77.27 \%$ respectively (Table 1 ).

Similar synergistic affect was observed when TRAIL expressing MSCs were co-cultured with Vorinostat treated MPM cell lines. MSC-TRAIL induced apoptosis in JU77 (48.73\%), CRL2081 (57.63\%) and ONE58(53.8\%). Combined treatment of Vorinostat and MSC-TRAIL significantly increased apoptosis to $77.7 \%$ in JU77, $90.93 \%$ in CRL2081 and $77.8 \%$ in ONE5 8 cells (Table 1).

Conclusion The combination of Vorinostat and recombinant TRAIL acts synergistically to induce apoptosis in malignant plural mesothelioma cells. Similar affect is observed with the combination of MSC-TRAIL and Vorinostat. This study indicates that Mesenchymal stem cells can be used as vectors for delivery of TRAIL and upon combination with Vorinostat, could be a potential therapy for malignant pleural mesothelioma.

\section{S128 REDUCTION OF LUNG METASTASIS BY ENGINEERED MESENCHYMAL STEM CELLS EXPRESSING SECRETED SOLUBLE TRAIL}

ZQY Yuan, KK Kolluri; University College London, London, UK

\subsection{6/thoraxjnl-2013-204457.135}

Bone marrow-derived mesenchymal stem cells (MSC) are promising tools for cancer therapy because they are able to home to and incorporate within tumour tissue. Tumour necrosis factor-related apoptosis-inducing ligand (TRAIL) is a pro-apoptotic protein that induces selective apoptosis of tumour cells, while sparing normal cells. Therefore it is expected that MSCs engineered to produce TRAIL would home to and kill cancer cells in a lung metastatic cancer model. Two lentiviral vectors were constructed to express the full-length (FL) TRAIL and a truncated soluble form (ILZ-sT), respectively. A secretion peptide and an isoleucine zipper (ILZ) peptide were added to the N-terminal of the soluble form to force its secreted expression and to enhance its trimerization. Human MSCs were transduced with viruses and both constructs produced soluble TRAIL into cell media that can rapidly induced apoptosis of cancer cells. However the ILZ-sT fusion construct expresses significantly higher level of soluble TRAIL, and causes better in vitro lung cancer cell (A549) killing than the FL one.

In coculture experiments both construct viruses transduced MSCs caused lung (A549), breast (MDAMB231), squamous (H357), and cervical (Hela) cancer cell apoptosis and death with similar efficiencies. A synergistic effect of cancer cell killing was observed for the combinational treatment of MSC-TRAIL cells with Saha, a histone deactylase inhibitor. When systemically delivered both MSC-FLT and MSC-ILZ-sT cells showed significant reduction of lung metastasis in a pulmonary metastasis murine model. Interestingly, ILZ-sT expressing cells demonstrated higher efficiency of metastasis reduction than FLT cells. These findings suggests that TRAIL expressing MSCs particularly ILZsT cells could be potentially developed as a therapy for lung metastasis diseases.

\section{S129 THE NATURAL HISTORY OF BRONCHIAL PRE-INVASIVE DISEASE}

JM Brown, G Hardavella, B Carroll, M Falzon, N Navani, PJ George, SM Janes; University College London Hospital, London, UK

\subsection{6/thoraxinl-2013-204457.136}

Background Bronchial pre-invasive lesions represent the earliest stages of the stepwise progression of squamous carcinogenesis, they predominantly affect the large airways and are readily detectable using autofluoresence bronchoscopy (AFB) however very little is known about the natural history of these lesions and no randomised data exists to determine whether intervention before progression to invasion improves outcome.

Methods A total of 94 patients with bronchial dysplasia were enrolled into an on-going surveillance cohort at University College London Hospital running prospectively since 1999. Lesions were biopsied longitudinally and kept under regular surveillance with AFB and low dose annual CT scanning until resolution or progression to invasive disease occurred. Retrospective analysis of lesional destiny was undertaken to determine the proportions of progressive vs. regressive lesions that occur in low grade dysplasia (LGD- squamous metaplasia, mild and moderate dysplasia) 
ZQY Yuan, KK Kolluri. S128: Reduction of lung metastasis by engineered Mesenchymal stem cells expressing secreted soluble TRAIL. Thorax 2013;63(Supp 3):A66. doi: 10.1136/thoraxjnl-2013204457.135

The correct author list should read as:

ZQ Yuan, K Kolluri, S M Janes; University College London, UK.

Thorax 2014;69:239. doi:10.1136/thoraxjnl-2013-204457.135.corr1 